\title{
Restricted neck mobility in children with chronic tension type headache: a blinded, controlled study
}

\author{
Daniel M. Fernández-Mayoralas • César Fernández-de-las-Peñas • \\ Domingo Palacios-Ceña $\cdot$ Irene Cantarero-Villanueva • \\ Carolina Fernández-Lao $\cdot$ Juan A. Pareja
}

Received: 23 March 2010/Accepted: 12 May 2010/Published online: 28 May 2010

(C) Springer-Verlag 2010

\begin{abstract}
The main purpose of this study was to analyze the differences in neck mobility between children with chronic tension type headache (CTTH) and healthy children, and to determine the influence of cervical mobility on headache intensity, frequency and duration. Fifty children, 13 boys and 37 girls (mean age $8.5 \pm 1.6$ years) with $\mathrm{CTTH}$ associated to peri-cranial tenderness (IHS 2.3.1) and 50 age- and sex matched children without headache (13 boys, 37 girls, mean age $8.5 \pm 1.8$ years, $P=0.955)$ participated. Cervical range of motion (CROM) was objectively assessed with a cervical goniometer by an assessor blinded to the children's condition. Children completed a headache diary for 4 weeks to confirm the diagnosis. Children with CTTH showed decreased CROM as compared to children without headache for flexion $(z=-6.170 ; P<0.001)$, extension $(z=-4.230 ; P<0.001)$, right $(z=-4.505$;
\end{abstract}

D. M. Fernández-Mayoralas · J. A. Pareja

Hospital Quirón de Madrid, Madrid, Spain

D. M. Fernández-Mayoralas

Centro "CADE", Madrid, Spain

C. Fernández-de-las-Peñas

Department of Physical Therapy, Occupational Therapy,

Rehabilitation and Physical Medicine, Universidad Rey Juan

Carlos, Alcorcón, Madrid, Spain

C. Fernández-de-las-Peñas

Esthesiology Laboratory of Universidad Rey Juan Carlos,

Alcorcón, Madrid, Spain

C. Fernández-de-las-Peñas

Department of Health Science and Technology,

Centre for Sensory-Motor Interaction (SMI),

Aalborg University, Aalborg, Denmark
$P<0.001)$ and left $(z=-4.768 ; P<0.001)$ lateralflexions, but not for rotation (right $z=-0.802$; $P=0.425 ;$ left $z=-1.254 ; P=0.213$ ) and also for total range of motion for flexion-extension $(z=-4.267$; $P<0.001)$ and lateral-flexion $(z=-4.801 ; P<0.001)$, but not for rotation $(z=-1.058 ; P=0.293)$. Within CTTH children, CROM was not correlated with headache intensity, frequency or duration. Additionally, age $(P>0.125)$ or gender $(P>0.250)$ did not influence CROM in either children with CTTH or without headache. Current results support the hypothesis that the cervical spine should be explored in children with headache. Further research is also needed to clearly define the potential role of the cervical spine in the genesis or maintenance of CTTH.

Keywords Tension-type headache .

Cervical range of motion $\cdot$ Cervical spine $\cdot$ Children

D. Palacios-Ceña

Department of Health Sciences II, Universidad Rey Juan Carlos, Madrid, Spain

I. Cantarero-Villanueva $\cdot$ C. Fernández-Lao

Department of Physical Therapy, Universidad Granada,

Granada, Spain

J. A. Pareja

Neurology Department, Hospital Fundación Alcorcón,

Madrid, Spain

C. Fernández-de-las-Peñas $(\varangle)$

Facultad de Ciencias de la Salud, Universidad Rey Juan Carlos, Avenida de Atenas s/n, 28922 Alcorcón, Madrid, Spain

e-mail: cesar.fernandez@urjc.es 


\section{Introduction}

Tension-type headache is the most common form of headache in both adults [1] and adolescents [2]. Different studies have reported an overall prevalence rate ranging from 5.5 to $26 \%$ in children between 6 and 12 years old [3-5]. In addition, tension type headache may cause substantial levels of disability for patients and their families as well as to the global society due to its very high prevalence in the general population [6].

Although there has been an increasing interest in the pathogenic mechanisms of tension type headache, pathoanatomical mechanisms remain inconclusive [7]. Previous studies have investigated pressure pain thresholds to further gain knowledge about pain mechanisms in children with headache $[8,9]$. These studies found that the cervical spine shows hyperalgesic responses in children with headache [8, 9]. In addition, Laimi et al. [10] showed that children with headache also suffered from neck pain [11], and that the presence of neck pain constitutes a negative prognostic factor for the headache. Therefore, these authors suggested that the neck should be considered in children with headache and that cervical evaluation is recommended when planning the treatment of these children [11].

In fact, cervical dysfunctions have been traditionally linked to different headaches. For instance, restricted neck mobility has been found in cervicogenic [12, 13], and chronic tension type headache (CTTH) [14], but not in migraine [15]. Scientific data referring to neck mobility in children with headache are scarce, even though we usually perceive in our clinical practice that children with this headache also show restricted neck mobility. Further, the only study which has analyzed neck mobility in children with headache did not find clear differences between migraine and episodic tension type headache [16]. The authors of this study recognized that their results may be related to the fact that a subgroup of children with tension type headache was included [16]. Due to the possible influence of the cervical spine in the development or maintenance of headache, further studies of neck mobility in children with tension type headache are required. To the best of the authors' knowledge, there is no study investigating differences in neck mobility between children with CTTH and healthy children. The aim of this study was to investigate the differences in cervical range of motion (CROM) between children with CTTH and children without headache, and the relationship between neck mobility and headache intensity, duration and frequency. We hypothesized that children with CTTH will show less CROM in all movements as compared to children without headache.

\section{Materials and methods}

\section{Subjects}

Consecutive children diagnosed with CTTH by an experienced neuropediatrist from the Neurology Department of Hospital Quirón were screened for eligibility criteria. To be included children had to describe the characteristics typical of CTTH associated with peri-cranial tenderness following the criteria of the International Headache Society (IHS 2.3.1) [17], bilateral location, pressing or tightening pain, $\mathrm{mild} /$ moderate intensity ( $\leq 6$ on a numerical pain rate scale) and no aggravation of headache during physical activity. Either photophobia or phonophobia, but not both, was permitted. No children reported vomiting or evident nausea during attacks. Other primary headaches and medicationoveruse headache were excluded [17]. No apparent evidence of secondary headache was present. None of the children had taken prophylactic drugs at the time of the study.

Additionally, age- and sex matched children without history of head or neck pain symptoms were recruited from volunteers who responded to a local announcement. Ethical approval was granted by local ethics committee (URJC/ FHA043). Informed consent was obtained from both children and parents and all procedures were conducted according to the declaration of Helsinki.

\section{Peri-cranial tenderness examination}

The total tenderness score (TTS) was used to assess pericranial tenderness [18]. Briefly, eight pairs of muscles and tendon insertions (i.e. masseter, temporal, frontal, trapezius, sternocleidomastoid, and suboccipital muscles, coronoid and mastoid processes) were palpated [18]. Manual palpation was conducted with small rotational movements of the assessors' second and third fingers for 4-5 s. Tenderness was scored on a four-point (0-3) scale at each location (local tenderness score). A TTS is calculated from the sum of scores from both sides (total maximum score 48) [18].

\section{Self-reported measures}

Children completed a headache diary for 4 weeks to complement the diagnosis [19]. An 11-point numerical pain rate scale [20] [NPRS; ranging from 0 (no pain), to 10 (maximum pain)] was used to assess headache intensity. The headache diary was used to calculate headache intensity (mean of the NPRS of the days with headache); headache frequency (calculated by dividing the number of days with headache by the number of the analyzed weeks, days/week) and headache duration (calculated by dividing 
the sum of the total hours of headache by the number of days with headache, $\mathrm{h} /$ day).

The beck depression inventory (BDI-II), a 21-item selfreport measure assessing affective, cognitive and somatic symptoms of depression, was also used for excluding children with high levels of depression [21]. Children, with the help of the parents, were asked to choose from a group of sentences which best described how they had been feeling in the preceding 2 weeks. For example, to assess sadness, they could choose either: "I do not feel sad", "I feel sad much of the time", "I am sad all the time", or "I am so sad or unhappy that I cannot stand it". The BDI-II has shown good internal consistency $(\alpha=0.86)$ with higher scores indicating higher levels of depression [22, 23].

\section{Cervical range of motion}

Neck mobility was assessed following the previous reported guidelines [14, 15]. A cervical goniometric device manufactured by Performance Attainment Associates (St. Paul, MN) was used. A recent study found an intratester reliability ranging from 0.87 to 0.96 and standard error of measurements between $2.3^{\circ}$ and $4.1^{\circ}$ in subjects with and without neck pain [24].

Neck mobility was assessed while sitting. It was recorded as the total range of motion for different types of movement, i.e. flexion/extension, lateral flexion, and rotation; as well as for half-cycles, namely movements in a single direction, i.e. flexion or extension, and right/left lateral-flexion, right/left rotation. For this purpose, all children were asked to sit comfortably on a chair with both feet flat on the floor, the hips and knees positioned at $90^{\circ}$ angles, and buttocks positioned against the back of the chair. Then, the goniometer was placed at the top of the head. Once the goniometer was set in neutral position, children were asked to move the head as far as possible in standard form forwards (flexion), backwards (extension), right and left lateral flexion, right and left rotation. Three measurements for each type of movement were recorded, and the mean was employed in the main statistical analysis. CROM was taken by an assessor blinded to the children's condition.

\section{Statistical analysis}

Data were analysed with the SPSS statistical package (14.0 version). Results are expressed as mean and 95\% confidence interval $(95 \% \mathrm{CI})$. The Kolmogorov-Smirnov test revealed that any variable showed normal distribution $(P<0.05)$; accordingly, non-parametric tests were used. Differences in neck mobility between groups were assessed with the Mann-Whitney $U$ test. Gender was included in the main analysis as a covariate. In addition, pairwise comparison between genders was also assessed with the MannWhitney $U$ test. The Spearman's rho $\left(r_{\mathrm{s}}\right)$ test was used to analyze the correlation between cervical mobility and headache intensity, frequency or duration in the patient group. The Spearman's rho $\left(r_{\mathrm{s}}\right)$ was again used to investigate the correlation between age and neck range of motion within each group. In general, a $P$ value less than 0.05 was considered statistically significant; however, when two related comparisons were conducted (flexion/extension, right/left lateral-flexions, and right/left rotations), the $P$ values were post hoc Bonferroni corrected and values less than 0.025 were used as threshold for significance.

\section{Results}

Eighty $(n=80)$ consecutive children presenting with headache between May 2009 and January 2010 were screened. Thirty $(38 \%)$ were excluded: migraine $(n=12)$, hemi-cranial headache $(n=5)$, higher levels of depression $(n=7)$ or anxiety $(n=6)$. Finally, a total of 50 children, 13 boys and 37 girls, aged 5-11 years (mean $8.5 \pm$ 1.6 years ) satisfied all the inclusion criteria (IHS 2.3.1, TTS $20 \pm 5$ ) and agreed to participate. In our sample, headache history was 2.1 years (95\% CI $1.8-2.4$ years), mean headache period per day was $4.2 \mathrm{~h}$ (95\% CI $3.5-$ $4.9 \mathrm{~h}$ ), the mean intensity per episode was 5.1 (95\% CI 4.8-5.4), and the number of days per week with headache was 4.2 (95\% CI 4.0-4.5 days/week). A significant positive correlation $\left(r_{\mathrm{s}}=0.284 ; P=0.046\right)$ between headache frequency and headache duration was found: the greater the frequency of the headache, the longer the duration. The BDI-II score was 3.7 (95\% CI 3.3-4.1). No significant correlations between headache parameters and BDI-II were found $(P>0.587)$.

In addition, 50 age- and sex matched children without headache, 13 boys and 37 girls, aged 5-11 years (mean $8.5 \pm 1.8$ years old $)$ also participated $(P=0.955)$.

Children with CTTH showed decreased CROM when compared to children without headache for flexion $(z=$ -6.170; $P<0.001)$, extension $(z=-4.230 ; P<0.001)$, right $(z=-4.505 ; P<0.001)$ and left $(z=-4.768$; $P<0.001$ ) lateral-flexions, but not for rotation (right $z=$ $-0.802 ; P=0.425$; left $z=-1.254 ; P=0.213$ ) and also for total range of motion for flexion-extension $(z=$ $-4.267 ; \quad P<0.001)$ and lateral-flexion $(z=-4.801$; $P<0.001)$, but not for rotation $(z=-1.058 ; P=0.293)$. Table 1 shows the CROM measurements for both groups.

Within CTTH children, CROM was not correlated with headache intensity, frequency or duration (Table 2). Additionally, age $(P>0.125)$ or gender $(P>0.250)$ did not influence CROM in children with CTTH or healthy 
Table 1 Range of motion for all cervical movements of each study group

CTTH chronic tension-type headache

Values are expressed in mean \pm standard deviation ( $95 \%$ confidence interval)

$P$ values come from the unpaired Student's $t$ test (a $P$ value less than 0.025 was considered statistically significant)

Table 2 Lineal negative correlations between headache clinical parameters and cervical range of motion within children with chronic tension type headache

\begin{tabular}{lccr}
\hline & CTTH group & Control group & $P$ value \\
\hline Flexion/extension & & & \\
Flexion & $55.4^{\circ} \pm 6.3^{\circ}\left(53.6^{\circ}-57.2^{\circ}\right)$ & $64.6^{\circ} \pm 5.8^{\circ}\left(62.9^{\circ}-66.3^{\circ}\right)$ & $<0.001$ \\
Extension & $69.9^{\circ} \pm 10.8^{\circ}\left(66.5^{\circ}-72.8^{\circ}\right)$ & $78.2^{\circ} \pm 7.4^{\circ}\left(76.1^{\circ}-80.3^{\circ}\right)$ & \\
Total & $125.1^{\circ} \pm 12.9^{\circ}\left(121.3^{\circ}-128.9^{\circ}\right)$ & $142.8^{\circ} \pm 9.7^{\circ}\left(140.0^{\circ}-145.6^{\circ}\right)$ & \\
Lateral flexion & & & \\
Right & $39.2^{\circ} \pm 5.9^{\circ}\left(37.4^{\circ}-40.8^{\circ}\right)$ & $48.4^{\circ} \pm 3.5^{\circ}\left(44.6^{\circ}-52.3^{\circ}\right)$ & $<0.001$ \\
Left & $40.8^{\circ} \pm 4.7^{\circ}\left(39.4^{\circ}-42.2^{\circ}\right)$ & $48.8^{\circ} \pm 2.3^{\circ}\left(45.2^{\circ}-52.4^{\circ}\right)$ & \\
Total & $80.0^{\circ} \pm 9.5^{\circ}\left(77.2^{\circ}-82.7^{\circ}\right)$ & $97.3^{\circ} \pm 6.2^{\circ}\left(89.8^{\circ}-104.7^{\circ}\right)$ & \\
Rotation & & & \\
Right & $77.6^{\circ} \pm 7.7^{\circ}\left(75.3^{\circ}-79.8^{\circ}\right)$ & $79.4^{\circ} \pm 9.9^{\circ}\left(75.6^{\circ}-83.0^{\circ}\right)$ & 0.425 \\
Left & $75.5^{\circ} \pm 8.8^{\circ}\left(72.9^{\circ}-78.1^{\circ}\right)$ & $78.4^{\circ} \pm 9.3^{\circ}\left(74.6^{\circ}-82.2^{\circ}\right)$ & 0.213 \\
Total & $153.1^{\circ} \pm 15.5^{\circ}\left(148.6^{\circ}-157.6^{\circ}\right)$ & $157.7^{\circ} \pm 16.1^{\circ}\left(150.3^{\circ}-165.1^{\circ}\right)$ & 0.293 \\
\hline
\end{tabular}

$r_{\mathrm{s}}$ rho de Spearman

\begin{tabular}{lll}
\hline Headache intensity & Headache duration & Headache frequency \\
\hline Cervical flexion & & \\
$r_{\mathrm{s}}=-0.248 ; P=0.089$ & $r_{\mathrm{s}}=-0.139 ; P=0.345$ & $r_{\mathrm{s}}=-0.039 ; P=0.792$ \\
Cervical extension & & \\
$r_{\mathrm{s}}=-0.220 ; P=0.133$ & $r_{\mathrm{s}}=-0.088 ; P=0.554$ & \\
Cervical right lateral-flexion & & $r_{\mathrm{s}}=-0.077 ; P=0.603$ \\
$r_{\mathrm{s}}=-0.056 ; P=0.705$ & $r_{\mathrm{s}}=-0.021 ; P=0.886$ & $r_{\mathrm{s}}=-0.102 ; P=0.492$ \\
Cervical left lateral-flexion & & \\
$r_{\mathrm{s}}=-0.089 ; P=0.545$ & $r_{\mathrm{s}}=-0.090 ; P=0.542$ & $r_{\mathrm{s}}=-0.183 ; P=0.214$ \\
Cervical right rotation & & \\
$r_{\mathrm{s}}=-0.143 ; P=0.331$ & $r_{\mathrm{s}}=-0.012 ; P=0.935$ & $r_{\mathrm{s}}=-0.031 ; P=0.835$ \\
Cervical left rotation & & $r_{\mathrm{s}}=-0.099 ; P=0.502$ \\
$r_{\mathrm{s}}=-0.019 ; P=0.898$ & &
\end{tabular}

control. Table 3 presents CROM on each group depending on gender.

\section{Discussion}

The results of the current study found that children with CTTH showed decreased CROM in flexion, extension and both lateral-flexions, but not rotation motions when compared to children without headache. Nevertheless, CROM was not correlated to headache intensity, frequency or duration, age or gender within the CTTH group.

The presence of limited range of motion in children with CTTH agrees with the results previously Fernández-de-las Peñas et al. [14] in CTTH adults, but disagrees with those found by Oksanen et al. [16] in children with episodic tension type headache. In fact, CROM reported by Oksanen et al. [16] for flexion, extension and lateral-flexions were lower than the values found in our study. Differences between both studies are probably related to the fact that Oksanen et al. [16] only included adolescents with episodic tension type headache, whereas we included children suffering from CTTH. It is possible that CROM is limited in the chronic, but not the episodic, form of tension type headache. This assumption would agree with the results reported by Fernández-de-las Peñas et al. [14] in adults with CTTH.

In a meta-analysis, Chen et al. [25] determined the following normative values for cervical mobility: flexion/ extension: $150^{\circ}-116^{\circ}$ (flexion $69^{\circ}-48^{\circ}$; extension $93^{\circ}-61^{\circ}$ ), lateral flexion: $108^{\circ}-76^{\circ}$ (side: $49^{\circ}-38^{\circ}$ ); rotation: $186^{\circ}$ $136^{\circ}$ (side: $93^{\circ}-70^{\circ}$ ). Based on these data, CROM of our CTTH children was in the lower $25 \%$ of the normative values, whereas the range of motion of children without headache was in the upper $75 \%$ of the normative values. If we considered that CROM decreases with age [26, 27], our results suggest that restricted $\mathrm{CROM}$ may be a feature of children with CTTH. In fact, neck mobility in children with CTTH was greater than that reported for adults with CTTH $\left(103.9^{\circ} \pm 25.7^{\circ}\right.$ flexion/extension; $67.1^{\circ} \pm 14.8^{\circ}$ lateralflexion; $116^{\circ} \pm 16.1^{\circ}$ rotation) [14].

An interesting finding was that children with CTTH had restricted neck mobility in flexion/extension and 
Table 3 Cervical range of motion in both groups depending on gender

CTTH chronic tension-type headache

Values are expressed in mean \pm standard deviation

\begin{tabular}{|c|c|c|c|c|}
\hline & \multicolumn{2}{|c|}{ Children with CTTH } & \multicolumn{2}{|c|}{ Healthy children } \\
\hline & Male $(n=13)$ & Female $(n=35)$ & Male & Female \\
\hline Cervical flexion & $53.8^{\circ} \pm 8.2^{\circ}$ & $56.0^{\circ} \pm 5.4^{\circ}$ & $65.4^{\circ} \pm 6.3^{\circ}$ & $64.3^{\circ} \pm 5.7^{\circ}$ \\
\hline Cervical extension & $67.9^{\circ} \pm 13.1^{\circ}$ & $70.4^{\circ} \pm 9.9^{\circ}$ & $78.1^{\circ} \pm 6.9^{\circ}$ & $78.2^{\circ} \pm 7.6^{\circ}$ \\
\hline Right lateral-flexion & $40.4^{\circ} \pm 5.6^{\circ}$ & $38.7^{\circ} \pm 5.9^{\circ}$ & $45.7^{\circ} \pm 12.0^{\circ}$ & $49.4^{\circ} \pm 14.1^{\circ}$ \\
\hline Left lateral-flexion & $41.5^{\circ} \pm 4.7^{\circ}$ & $40.5^{\circ} \pm 4.8^{\circ}$ & $46.9^{\circ} \pm 10.1^{\circ}$ & $49.5^{\circ} \pm 13.5^{\circ}$ \\
\hline Right rotation & $78.3^{\circ} \pm 6.7^{\circ}$ & $77.3^{\circ} \pm 8.1^{\circ}$ & $80.5^{\circ} \pm 10.9^{\circ}$ & $78.9^{\circ} \pm 13.7^{\circ}$ \\
\hline Left rotation & $74.2^{\circ} \pm 11.3^{\circ}$ & $76.0^{\circ} \pm 7.9^{\circ}$ & $79.6^{\circ} \pm 10.7^{\circ}$ & $78.0^{\circ} \pm 14.2^{\circ}$ \\
\hline
\end{tabular}

lateral-flexion, but not rotation motion. This finding may be related to shortening of particular muscles or the presence of myofascial trigger points in some muscles. For instance, shortening or trigger points in the splenius capitis muscle may contribute to neck flexion restriction, whereas sternocleidomastoid muscle trigger points or shortening may be related to cervical extension restriction. Additionally, it is possible that the differences in cervical biomechanical aspects may be related for CTTH in children. Future studies evaluating shortening and the presence of myofascial trigger points in the neck musculature are needed to further elucidate the role of these muscles in children with CTTH.

The presence of restricted neck mobility further support the hypothesis of Laimi et al. [11] that the cervical spine should be explored in children with headache and that cervical intervention may be recommended when planning the treatment of these children. In fact, restricted neck mobility may be due to neck pain, which constitutes a negative prognostic factor for the course of headache [10]. This hypothesis may be related to the fact that CROM did not correlate with clinical variables concerning the intensity and the temporal profile of the headache which suggests that restricted cervical mobility is a consequence rather than a causative factor of pain. The relevance of the cervical spine for head pain is explained by the convergence of afferent nerve fibres from the trigeminal and cervical regions within the trigemino-cervical nucleus caudalis [28]. It would be possible that nociceptive afferences originating in cervical structures may influence the excitability of the trigeminal nucleus caudalis, further promoting the maintenance of headache. Therefore, considering the restricted CROM in our sample of children with CTTH, it may be hypothesized that treatment directed at the cervical spine may enhance outcomes of children with CTTH. Further studies are required to define the connections between the cervical spine and headache in children. Future clinical trials should investigate the effects of treatment directed at the cervical spine in children with CTTH.

We should recognize some limitations of the current study. First, we included children from a specialized neurologic clinic. Therefore, our sample may not represent the characteristics of the general headache children population. Therefore, extrapolation of current results should be conducted with caution. Population-based epidemiological studies with larger samples are needed for permitting a more generalized interpretation of the current results. Secondly, this was a cross-sectional study, therefore any causal relationship cannot be indicated. Longitudinal studies are required to define the role of the cervical spine in the development or maintenance of pain in children with CTTH.

\section{Conclusion}

Children with CTTH associated with peri-cranial tenderness (IHS 2.3.1) showed lesser CROM than children without headache. Neck mobility was not associated with headache intensity, frequency or duration and was not influenced by age or gender. Current results would support the hypothesis that the cervical spine should be explored in children with CTTH. Further research is needed to define the potential role of the neck in the genesis or maintenance of CTTH in children.

Conflict of interest None.

\section{References}

1. Andlin-Sobocki P, Jonsson B, Wittchen HU, Olesen J (2005) Cost of disorders of the brain in Europe. Eur J Neurol 12(Suppl 1): $1-27$

2. Unalp A, Dirik E, Kurul S (2007) Prevalence and clinical findings of migraine and tension-type headache in adolescents. Pediatr Int 49:943-949

3. Ayatollahi SM, Khosravi A (2006) Prevalence of migraine and tension-type headache in primary-school children in Shiraz. East Mediterr Health J 12:809-817

4. Laurell K, Larsson B, Eeg-Olofsson O (2004) Prevalence of headache in Swedish school-children, with a focus on tensiontype headache. Cephalalgia 24:380-388

5. Zwart JA, Dyb G, Holmen TL, Stovner LJ, Sand T (2004) The prevalence of migraine and tension-type headaches among adolescents in Norway: the Nord-Trøndelag Health Study 
(Head-HUNT-Youth), a population-based epidemiological study. Cephalalgia 24:373-379

6. Stovner L, Hagen K, Jensen R, Katsarava Z, Lipton R, Scher A, Steiner T, Zwart JA (2007) The global burden of headache: a documentation of headache prevalence and disability worldwide. Cephalalgia 27:193-210

7. Fernández-de-las-Peñas C, Schoenen J (2009) Chronic tension type headache: what's new? Curr Opin Neurol 22:254-261

8. Anttila P, Metsahonkala L, Mikkelsson M et al (2002) Muscle tenderness in pericranial and neck-shoulder region in children with headache: a controlled study. Cephalalgia 22:340-344

9. Metsahonkala L, Anttila P, Laimi K et al (2006) Extra-cephalic tenderness and pressure pain threshold in children with headache. Eur J Pain 10:581-585

10. Laimi K, Vahlberg T, Salminen J, Metsähonkala L, Mikkelsson M, Anttila P, Aromaa M, Sillanpää M (2007) Does neck pain determine the outcome of adolescent headache? Cephalalgia 27:244253

11. Laimi K, Salminen JJ, Metsähonkala L, Vahlberg T, Mikkelsson M, Anttila P, Aromaa M, Rautava P, Suominen S, Liljeström MR, Sillanpää M (2007) Characteristics of neck pain associated with adolescent headache. Cephalalgia 27:1244-1254

12. Jull G, Amiri M, Bullock-Saxton J, Darnell R, Lander C (2007) Cervical musculoskeletal impairment in frequent intermittent headache-part 1: subjects with single headaches. Cephalalgia 27:793-802

13. Zito G, Jull G, Story I (2006) Clinical tests of musculoskeletal dysfunction in the diagnosis of cervicogenic headache. Manual Ther 11:118-129

14. Fernández-de-las-Peñas $\mathrm{C}$, Alonso Blanco $\mathrm{C}$, Cuadrado ML, Pareja JA (2006) Forward head posture and neck mobility in chronic tension type headache: a blinded, controlled study. Cephalalgia 26:314-319

15. Fernández-de-las-Peñas C, Cuadrado ML, Pareja JA (2006) Myofascial trigger points, neck mobility and forward head posture in unilateral migraine. Cephalalgia 26:1061-1070

16. Oksanen A, Metsähonkala L, Viander S, Jäppilä E, Aromaa M, Anttila P, Salminen J, Sillanpää M (2006) Strength and mobility of the neck-shoulder region in adolescent headache. Physiother Theory Pract 22:163-174

17. IHS: Headache Classification Subcommittee of the International Headache Society (2004) The International Classification of Headache Disorders, 2nd edn Cephalalgia 24 (suppl 1):9-160

18. Langemark M, Olesen J (1987) Pericranial tenderness in tension headache: a blind controlled study. Cephalalgia 7:245-249

19. Phillip D, Lyngberg AC, Jensen R (2007) Assessment of headache diagnosis: a comparative population study of a clinical interview with a diagnostic headache diary. Cephalalgia 27:1-8

20. Jensen MP, Turner JA, Romano JM, Fisher L (1999) Comparative reliability and validity of chronic pain intensity measures. Pain 83:157-162

21. Beck AT, Steer RA, Brown GK (1996) Beck depression inventory. 2nd edn, The Psychological Corporation. San Antonio

22. Beck AT, Steer RA, Garbin MG (1988) Psychometric properties of the Beck Depression Inventory: twenty-five years of evaluation. Clin Psychol Rev 8:77-100

23. VanVoorhis WCR, Blumentritt TL (2007) Psychometric properties of the beck depression inventory-II in a clinically-identified sample of Mexican American Adolescents. J Child Family Studies, pp 789-798

24. Fletcher JP, Bandy WD (2008) Intrarater reliability of CROM measurement of cervical spine active range of motion in persons with and without neck pain. J Orthop Sports Phys Ther 38:640645

25. Chen J, Solinger AB, Poncet JF, Lantz CA (1999) Meta-analysis of normative cervical motion. Spine 24:1571-1578

26. Netzer O, Paine VG (1993) Effects of age and gender on functional rotation and lateral movements of the neck and back. Gerontology 39:320-326

27. Dvorak J, Antinnes JA, Panjabi M, Loustalot D, Bonomo M (1992) Age and gender related normal motion of the cervical spine. Spine 17(Suppl 10):S393-S398

28. Mørch CD, Hu J, Arendt-Nielsen L, Sessle B (2009) Convergence of cutaneous, musculo-skeletal, dural and visceral afferents onto nociceptive neurons in the first cervical dorsal horn. Eur J Neuroscience 26:142-154 